\title{
Neutrino- ${ }^{12} \mathrm{C}$ scattering in the $a b$ initio shell model with a realistic three-body interaction
}

\author{
A. C. Hayes ${ }^{1}$, P. Navrátil ${ }^{2}$ and J. P. Vary ${ }^{3}$ \\ ${ }^{1}$ Theoretical Division, Los Alamos National Laboratory, Los Alamos, New Mexico 87545 \\ ${ }^{2}$ Lawrence Livermore National Laboratory, L-414, P.O. Box 808, Livermore, CA 94551 \\ ${ }^{3}$ Department of Physics and Astronomy, Iowa State University, Ames, IA 50011
}

\begin{abstract}
We investigate cross sections for neutrino- ${ }^{12} \mathrm{C}$ exclusive scattering and for muon capture on ${ }^{12} \mathrm{C}$ using wave functions obtained in the $a b$ initio no-core shell model. In our parameter-free calculations with basis spaces up to the $6 \hbar \Omega$ we show that realistic nucleon-nucleon interactions, like e.g. the CD-Bonn, under predict the experimental cross sections by more than a factor of two. By including a realistic three-body interaction, Tucson-Melbourne $\mathrm{TM}^{\prime}(99)$, the cross sections are enhanced significantly and a much better agreement with experiment is achieved. At the same time, the $\mathrm{TM}^{\prime}(99)$ interaction improves the calculated level ordering in ${ }^{12} \mathrm{C}$. The comparison between the CD-Bonn and the three-body calculations provides strong confirmation for the need to include a realistic three-body interaction to account for the spin-orbit strength in $p$-shell nuclei.
\end{abstract}

PACS numbers: 25.30.Pt, 21.60.Cs, 21.30.Fe, 27.20.+n

The Gamow-Teller (GT) transition from the ground state of ${ }^{12} \mathrm{C}$ to the $1^{+} \mathrm{T}=1$ isobar triplet $\left({ }^{12} \mathrm{~B}_{\text {g.s. }},{ }^{12} \mathrm{C}(15.11\right.$ $\mathrm{MeV}),{ }^{12} \mathrm{~N}_{\text {g.s. }}$.) is a very sensitive test of nuclear structure models for mass 12 and, particularly, of the strength of the spin-orbit interaction. The two most common $p$-shell approximations for the structure of the ground state of ${ }^{12} \mathrm{C}((\mathrm{a})$ the $p$-shell equivalent of a $\mathrm{L}=0 \mathrm{~S}=0$ three alpha-cluster structure and (b) the closed $p_{3 / 2}$ shell structure) give very different (indeed opposite) predictions for the $\mathrm{B}(\mathrm{GT})$ strength to $\mathrm{T}=11^{+}$triplet. In the $p$-shell alpha-cluster limit the ground state of carbon has good SU(4) symmetry [444] and the Gamow-Teller transition is forbidden because there does not exist a $1^{+} \mathrm{T}=1$ state with [444] symmetry and $\sigma \tau$ operator cannot change SU(4) symmetry. This translates into an exact cancellation between the different $p_{1 / 2}$ and $p_{3 / 2}$ transition amplitudes. The observed transition strength requires the inclusion of higher $\mathrm{SU}(4)$ components in the wave functions and the breaking of the cancellation is quite sensitive to the assumed spin-orbit interaction. In the the $j j$-coupling limit, where one assumes that the ground state of ${ }^{12} \mathrm{C}$ is described by a closed $p_{3 / 2}$ shell, the transition to the $\mathrm{T}=11^{+}$state is pure $p_{3 / 2} \rightarrow p_{1 / 2}$. No cancellations between different transition amplitudes are allowed and the transition strength is over estimated by almost a factor of 6 . When RPA correlations are included in the initial and final states the situation improves somewhat, but the transition remains over-estimated by about a factor of 4 [1, 2]. The strong contrast between the predictions of the pure $j j$-coupling and the pure $\mathrm{SU}(4)$ limits makes this Gamow-Teller transition an ideal test case for the strength of the spin-orbit interaction and for model wave functions of mass 12 .

In this letter we present the predictions of no-core shell model (NCSM) [3] calculations of ${ }^{12} \mathrm{C}$ for the $\mathrm{T}=11^{+}$ transition in ${ }^{12} \mathrm{C}$. We examine inelastic electron scattering to the $15.11 \mathrm{MeV}$ state of ${ }^{12} \mathrm{C}$, muon capture to the ground state of ${ }^{12} \mathrm{~B}$, and neutrino scattering to the ground state of ${ }^{12} \mathrm{~N}$. These different electroweak reactions probe different momentum transfers and comparisons between theory and experiment allow us to test the convergence of the no-core shell model with increasing basis size, up to $6 \hbar \Omega$. We also investigate the contributions of a three-nucleon force since it is now well established [4, 5, 6] that realistic nucleon-nucleon interactions alone account for only half the observed $p$-shell splitting, while the rest arises form two-pion exchange between three or more nucleons. In the present calculations we include a realistic chiral-symmetry-based three-nucleon interaction (TNI), Tucson-Melbourne $\operatorname{TM}^{\prime}(99)$ ].

A detailed description of the NCSM approach was presented, e.g. in Refs. 3, 8]. Here, we simply present extensions and modifications needed when a genuine TNI is included. The starting Hamiltonian is $H_{A}=\frac{1}{A} \sum_{i<j} \frac{\left(\vec{p}_{i}-\vec{p}_{j}\right)^{2}}{2 m}+$ $\sum_{i<j}^{A} V_{\mathrm{NN}, i j}+\sum_{i<j<k}^{A} V_{\mathrm{NNN}, i j k}$, where $V_{\mathrm{NN}, i j}$ is the nucleon-nucleon (NN) interaction and $V_{\mathrm{NNN}, i j k}$ is the TNI. We employ a large but finite harmonic-oscillator (HO) basis. Due to properties of the realistic nuclear interaction we have to derive an effective interaction appropriate for the selected finite basis space. To facilitate this, we modify the Hamiltonian by adding to it the center-of-mass (CM) HO Hamiltonian $H_{\mathrm{CM}}=T_{\mathrm{CM}}+U_{\mathrm{CM}}$, where $U_{\mathrm{CM}}=$ $\frac{1}{2} A m \Omega^{2} \vec{R}^{2}, \vec{R}=\frac{1}{A} \sum_{i=1}^{A} \vec{r}_{i}$. The effect of the HO CM Hamiltonian will later be subtracted in the final many-body calculation. The modified Hamiltonian can be cast into the form $H_{A}^{\Omega}=H_{A}+H_{\mathrm{CM}}=\sum_{i=1}^{A} h_{i}+\sum_{i<j}^{A} V_{i j}^{\Omega, A}+$ $\sum_{i<j<k}^{A} V_{\mathrm{NNN}, i j k}$, where $h_{i}=\frac{\vec{p}_{i}^{2}}{2 m}+\frac{1}{2} m \Omega^{2} \vec{r}_{i}^{2}$ and $V_{i j}^{\Omega, A}=V_{\mathrm{NN}, i j}-\frac{m \Omega^{2}}{2 A}\left(\vec{r}_{i}-\vec{r}_{j}\right)^{2}$. Next we divide the $A$-nucleon infinite HO basis space into the finite active space $(P)$ comprising all states up to $N_{\max }$ HO excitations above the unperturbed ground state and the excluded spaces $(Q=1-P)$. The basic idea of the NCSM approach is to apply a unitary transformation on the modified Hamiltonian, $e^{-S} H_{A}^{\Omega} e^{S}$ such that $Q e^{-S} H_{A}^{\Omega} e^{S} P=0$. If such a transformation 
is found, the effective Hamiltonian that exactly reproduces a subset of eigenstates of the full space Hamiltonian is given by $H_{\text {eff }}=P e^{-S} H_{A}^{\Omega} e^{S} P$. This effective Hamiltonian contains up to $A$-body terms and it is essentially as difficult to construct it as to solve the full problem. Therefore, we apply this approach with a cluster approximation. When a genuine TNI is considered, the simplest cluster approximation produces a three-body effective interaction. The NCSM calculation is then performed in four steps:

(i) We solve a three-nucleon system for all possible three-nucleon channels with the Hamiltonian $H_{A}^{\Omega}$,i.e., using $h_{1}+h_{2}+h_{3}+V_{12}^{\Omega, A}+V_{13}^{\Omega, A}+V_{23}^{\Omega, A}+V_{\mathrm{NNN}, 123}$. Consequently, the three nucleons feel a pseudo-mean field of the spectator nucleons generated by the HO CM potential. It is necessary to separate the three-body effective interaction contributions from the TNI and from the two-nucleon interaction. Therefore, we need to find three-nucleon solutions for the Hamiltonian with and without the $V_{\mathrm{NNN}, 123} \mathrm{TNI}$ term. The three-nucleon solutions are obtained by procedures described in Refs. 9] (without TNI) and 10] (with TNI).

(ii) We construct the unitary transformation corresponding to the choice of the active basis space $P$ from the three-nucleon solutions using the Lee-Suzuki procedure 11, 12]. The three-body effective interaction is then obtained as $V_{3 \mathrm{eff}, 123}^{\mathrm{NN}+\mathrm{NNN}}=P\left[e^{-S_{\mathrm{NN}+\mathrm{NNN}}}\left(h_{1}+h_{2}+h_{3}+V_{12}^{\Omega, A}+V_{13}^{\Omega, A}+V_{23}^{\Omega, A}+V_{\mathrm{NNN}, 123}\right) e^{S_{\mathrm{NN}+\mathrm{NNN}}}-\left(h_{1}+h_{2}+h_{3}\right)\right] P$ and $V_{3 \mathrm{eff}, 123}^{\mathrm{NN}}=P\left[e^{-S_{\mathrm{NN}}}\left(h_{1}+h_{2}+h_{3}+V_{12}^{\Omega, A}+V_{13}^{\Omega, A}+V_{23}^{\Omega, A}\right) e^{S_{\mathrm{NN}}}-\left(h_{1}+h_{2}+h_{3}\right)\right] P$. The three-body effective interaction contribution from the TNI is then defined as $V_{3 \mathrm{eff}, 123}^{\mathrm{NNN}} \equiv V_{3 \mathrm{eff}, 123}^{\mathrm{NN}+\mathrm{NNN}}-V_{3 \mathrm{eff}, 123}^{\mathrm{NN}}$.

(iii) As the three-body effective interactions are derived in the Jacobi-coordinate HO basis but the $A=12$ calculations will be performed in a Cartesian-coordinate single-particle Slater-determinant m-scheme basis, we need to perform a suitable transformation of the interactions. This transformation is a generalization of the well-known transformation on the two-body level that depends on HO Brody-Moshinsky brackets.

(iv) We solve the Schrödinger equation for the $A=12$ nucleon system using the Hamiltonian $H_{A \text {,eff }}^{\Omega}=\sum_{i=1}^{A} h_{i}+$ $\frac{1}{A-2} \sum_{i<j<k}^{A} V_{3 \mathrm{eff}, i j k}^{\mathrm{NN}}+\sum_{i<j<k}^{A} V_{3 \mathrm{eff}, i j k}^{\mathrm{NNN}}$, where the $\frac{1}{A-2}$ factor takes care of overcounting the contribution from the two-nucleon interaction. At this point we also subtract the $H_{\mathrm{CM}}$. The $A=12$ nucleon calculation is then performed using the Many-Fermion Dynamics shell model code [13] generalized to handle three-body interactions. Eventually, the transition densities are computed that serve as an input for evaluating our selected observables.

Detailed ${ }^{12} \mathrm{C}$ NCSM calculations using realistic two-nucleon interactions were reported in Ref. [3]. Here we extend those calculations by including the TNI and reach the $4 \hbar \Omega(6 \hbar \Omega)$ basis in calculations with (without) the TNI. In Table \we summarize some of our results. In general, in addition to increase of binding energy, we observe a substantial sensitivity of the low-lying spectra to the presence of the TNI and a trend toward level-ordering and level-spacing improvement in comparison to experiment. The sensitivity is the largest for states where the spin-orbit interaction strength is known to play a role. Note the correct ordering of the $1^{+} 0 \leftrightarrow 4^{+} 0$ states and ordering and spacing improvement of the lowest $\mathrm{T}=1$ states.

The significant increase in the spin-orbit splitting obtained from the inclusion of the TNI is seen most strikingly in the predicted cross sections to the $\mathrm{T}=11^{+}$states. In all our electron scattering and weak interactions results here, only one-body currents are included and the bare operators are used. Dubach and Haxton [17] have shown that at high-momentum-transfers it is necessary to include two-body meson-exchange currents to describe the transverse magnetic electron scattering form factor for excitation of the $15.11 \mathrm{MeV}$ state. However, a reasonable description of the form factor up to momentum transfers of about $200 \mathrm{MeV} / \mathrm{c}$ can be obtained with a one-body current.

Figure 1 1shows a comparison between the form factors predicted by the NCSM and experiment. The experimental data are represented by the black circles, which represent a fit to the data assuming only a one-body current obtained by Dubach and Haxton [17]. The theoretical curves shown are NCSM results for $2 \hbar \Omega, 4 \hbar \Omega, 6 \hbar \Omega$ using the CD-Bonn NN interaction and a $4 \hbar \Omega$ calculation using the $\mathrm{AV}^{\prime}$ plus the $\mathrm{TM}^{\prime}(99)$ realistic TNI. The qualitative features of our results are seen by looking at the height of the first maximum and the position of the minimum. With two-body interactions alone, the change in transition form factor from $4 \hbar \Omega$ to $6 \hbar \Omega$ is small compared to the differences between theory and experiment. The magnitude of the form factor is too low and minimum occurs too far out in momentum. When the TNI is included a significant improvement is seen in both the shape and magnitude of the theoretical form factor. The magnitude of the form factor up to the first maximum is close but somewhat lower than experiment. The shape of the form factor is also improved but it is still stretched out too far in momentum. Comparing the $4 \hbar \Omega$-body calculation with the $4 \hbar \Omega$ CD-Bonn calculation the magnitude of the form factor at the peak has increased by about $75 \%$, and the position of the first minimum has shifted from $q_{\min } \sim 400 \mathrm{MeV} / \mathrm{c}$ to $360 \mathrm{MeV} / \mathrm{c}$. This improvement is almost entirely due to the improved strength of the spin-orbit splitting when the TNI is included.

The 15.11 MeV state was included in the fit to the $p$-shell interaction by Cohen and Kurath 18] (CK), and the CK interaction probably represents the best description of this transition using a globally fitted $p$-shell interaction. Our $\mathrm{AV}^{\prime}+\mathrm{TM}^{\prime}(99)$ form factor agrees well with the CK prediction, when for consistency between the two calculations, we use $\mathrm{b}=1.663 \mathrm{fm}$.

The conclusion drawn from the transverse magnetic form factor results is further supported by our $\mathrm{B}\left(\mathrm{M} 1 ; 1^{+} 1 \rightarrow\right.$ $0^{+} 0$ ) results presented in Table $\$ and Fig. 2 The calculations with 2-body forces show saturation and underpredict 
the experiment by almost a factor of three. By including the TNI, the B(M1) value increases dramatically. We fully expect that further increases in the basis size will produce results with TNI close to experiment. For smaller basis sizes, effective transition operators may be important and work in this direction is underway.

Table 1 shows the comparison between the theoretical and experimental neutrino scattering cross section for the same selections of Hamiltonians and basis spaces. These results show a similar trend to the electron scattering results above. In this case, the neutrino spectrum for electron neutrinos from decay-at-rest (DAR) of the pion peaks around $30 \mathrm{MeV}$ and the average momentum transfer is about $40 \mathrm{MeV} / \mathrm{c}$. The CD-Bonn interaction (without TNI) results indicate an approach to convergence by $6 \hbar \Omega$ but experiment is under-predicted by about a factor of 2.4. When the TNI is included with the AV8' interaction the predicted cross section is only $30 \%$ lower than experiment. Based on the similarity of trends with the electron scattering results, we anticipate that when the model space is eventually expanded to $6 \hbar \Omega$ theory would be within $15 \%$ of experiment. The substitution of AV8' for CD-Bonn in the calculations with TNI is expected to be of minor consequence.

Muon capture involves a higher momentum transfer than the $\left(\nu_{e}, e^{-}\right)$reaction and the average momentum transfer is $q \sim 100 \mathrm{MeV} / \mathrm{c}$. By $6 \hbar \Omega$ the CD-Bonn calculations show signs of converging yet experiment is underestimated a factor of 2.6. The inclusion of the TNI shows a significant improvement and, for $4 \hbar \Omega$, theory is $34 \%$ lower than experiment. Again extrapolating using the trends of the inelastic electron scattering results suggests that a $6 \hbar \Omega$ calculation that included a realistic TNI would come within $20 \%$ of experiment.

The $\left(\nu_{\mu}, \mu^{-}\right)$neutrino cross section to ${ }^{12} \mathrm{~N}_{\text {g.s. }}$ corresponds to the LSND muon neutrinos from decay-in-flight (DIF) of the pion. This spectrum involves neutrinos up to about $250 \mathrm{MeV}$, with a average neutrino energy of about 150 $\mathrm{MeV}$ and an average momentum transfer of about $200 \mathrm{MeV} / \mathrm{c}$. In this case the $6 \hbar \Omega \mathrm{CD}$-Bonn calculation is off by a factor of 1.8 compared with experiment. The $4 \hbar \Omega$ calculation that includes the 3 -body $\operatorname{TM}^{\prime}(99)$ interaction is, in fact, in agreement with experiment. However, based on the trends established above, this suggests that a larger model space may over-predict experiment. Examining the elastic scattering form factor suggests that the problem lies in the fact that at $200 \mathrm{MeV} / \mathrm{c}$ the predicted form factor is too large. Of course, as the model space is increased we expect the form factor to be shifted down in momentum.

In conclusion, the transition from the ${ }^{12} \mathrm{C}_{\text {g.s. }}$. to the $\mathrm{T}=11^{+}$states in mass 12 is very sensitive to the strength of the spin orbit interaction. We have investigated neutrino- ${ }^{12} \mathrm{C}$ exclusive cross sections and muon capture on ${ }^{12} \mathrm{C}$ as well as inelastic electron scattering using wave functions obtained in the $a b$ initio NCSM. In our parameter-free calculations with basis spaces up to $6 \hbar \Omega$ we show that realistic NN interactions under predict the experimental weak interaction cross sections by more than a factor of two. At high momentum transfers around $q \sim 200 \mathrm{MeV} / \mathrm{c}$ the electron scattering form factor is over-predicted and the position of the predicted minimum to close to $400 \mathrm{MeV} / \mathrm{c}$, compared to the experimental minimum at $q \sim 260 \mathrm{MeV} / \mathrm{c}$. By including a realistic TNI the weak interaction cross sections are enhanced significantly which considerably improves agreement with experiment. The shape of the electron scattering form factor is also significantly improved, but the predicted form factor still peaks at too large a momentum transfer and is too large at the momentum transfers relevant to the LSND DIF cross section. The difference between the observed and predicted shape for the (e,e') form factors and the very different momentum transfers involved in the three weak processes examined here imply that a single experiment/theory scale factor cannot be defined for all three. The comparison between the CD-Bonn and the three-body calculations discussed here provide a strong confirmation of the need to include a realistic three-body interaction to account for the spin-orbit strength in $p$-shell nuclei.

This work was performed in part under the auspices of the U. S. Department of Energy by the University of California, Lawrence Livermore National Laboratory under contract No. W-7405-Eng-48. This work was also supported in part by USDOE DE-FG02-87ER40371.

[1] E. Kolbe, K. Langanke, and P. Vogel, Nucl. Phys. A652,91, (1999)

[2] A. C. Hayes and I. S. Towner, Phys. Rev. C 61, 044603 (2000).

[3] P. Navrátil, J. P. Vary and B. R. Barrett, Phys. Rev. Lett. 84, 5728 (2000); Phys. Rev. C 62, 054311 (2000).

[4] S. C. Pieper and V. R. Pandharipande, Phys. Rev. Lett. 70, 2541 (1999).

[5] S. C. Pieper, V. R. Pandharipande, R. B. Wirginga and J. Carlson, Phys. Rev. C 64, 014001 (2001).

[6] R. B. Wiringa and S. C. Pieper, Phys. Rev. Lett. 89, 182501 (2002).

[7] S. A. Coon and H. K. Han, Few-Body Systems 30, 131 (2001).

[8] P. Navrátil and W. E. Ormand, Phys. Rev. Lett. 88, 152502 (2002).

[9] P. Navrátil, G. P. Kamuntavičius and B.R. Barrett, Phys. Rev. C 61, 044001 (2000).

[10] D. C. J. Marsden, P. Navrátil, S. A. Coon and B. R. Barrett, Phys. Rev. C 66, 044007 (2002).

[11] K. Suzuki and S. Y. Lee, Prog. Theor. Phys. 64, 2091 (1980).

[12] K. Suzuki and R. Okamoto, Prog. Theor. Phys. 92, 1045 (1994).

[13] J. P. Vary, "The Many-Fermion-Dynamics Shell-Model Code", Iowa State University (1992) (unpublished). 


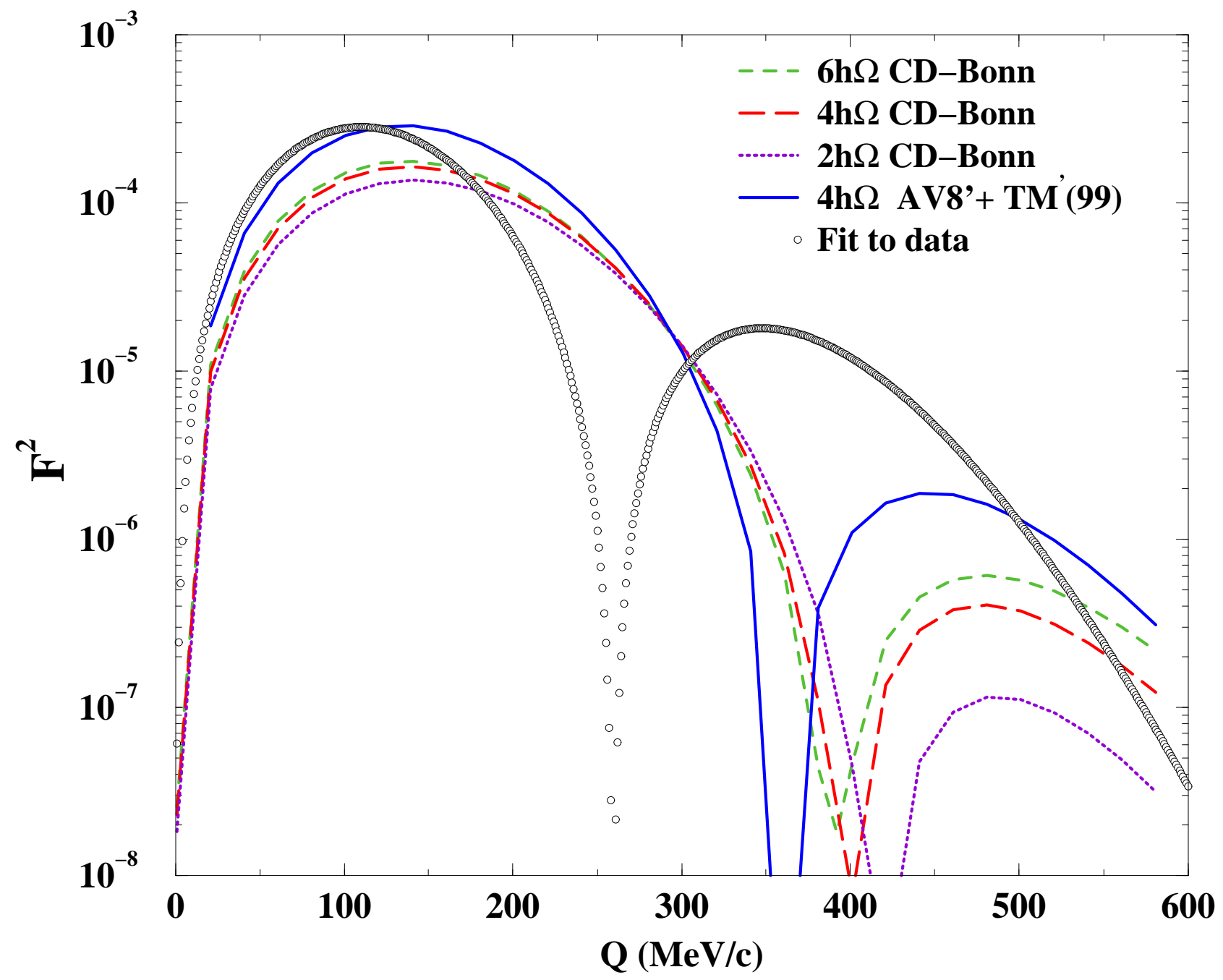

FIG. 1: The transverse magnetic electron scattering form factor for the $15.11 \mathrm{MeV} \mathrm{T}=11^{+}$state in ${ }^{12} \mathrm{C}$.

[14] B. S. Pudliner et al. Phys. Rev. C 56, 1720 (1997).

[15] R. Machleidt, F. Sammarruca and Y. Song, Phys. Rev. C 53, 1483 (1996).

[16] F. Ajzenberg-Selove, Nucl. Phys. A 506, 1 (1990).

[17] J. Dubach and W. C. Haxton, Phys. Rev. Lett. 41, 1453 (1978).

[18] S. Cohen and D. Kurath, Nucl. Phys. 73, 1 (1965).

[19] LSND collaboration, L. B. Auerbach et al., Phys. Rev. C 64, 065501 (2001).

[20] LSND collaboration, L. B. Auerbach et al., Phys. Rev. C 66, 015501 (2002).

[21] G. H. Miller et al., Phys. Lett. 41B, 50 (1972); M. Giffon et al., Phys. Rev. C 24, 241 (1981). 


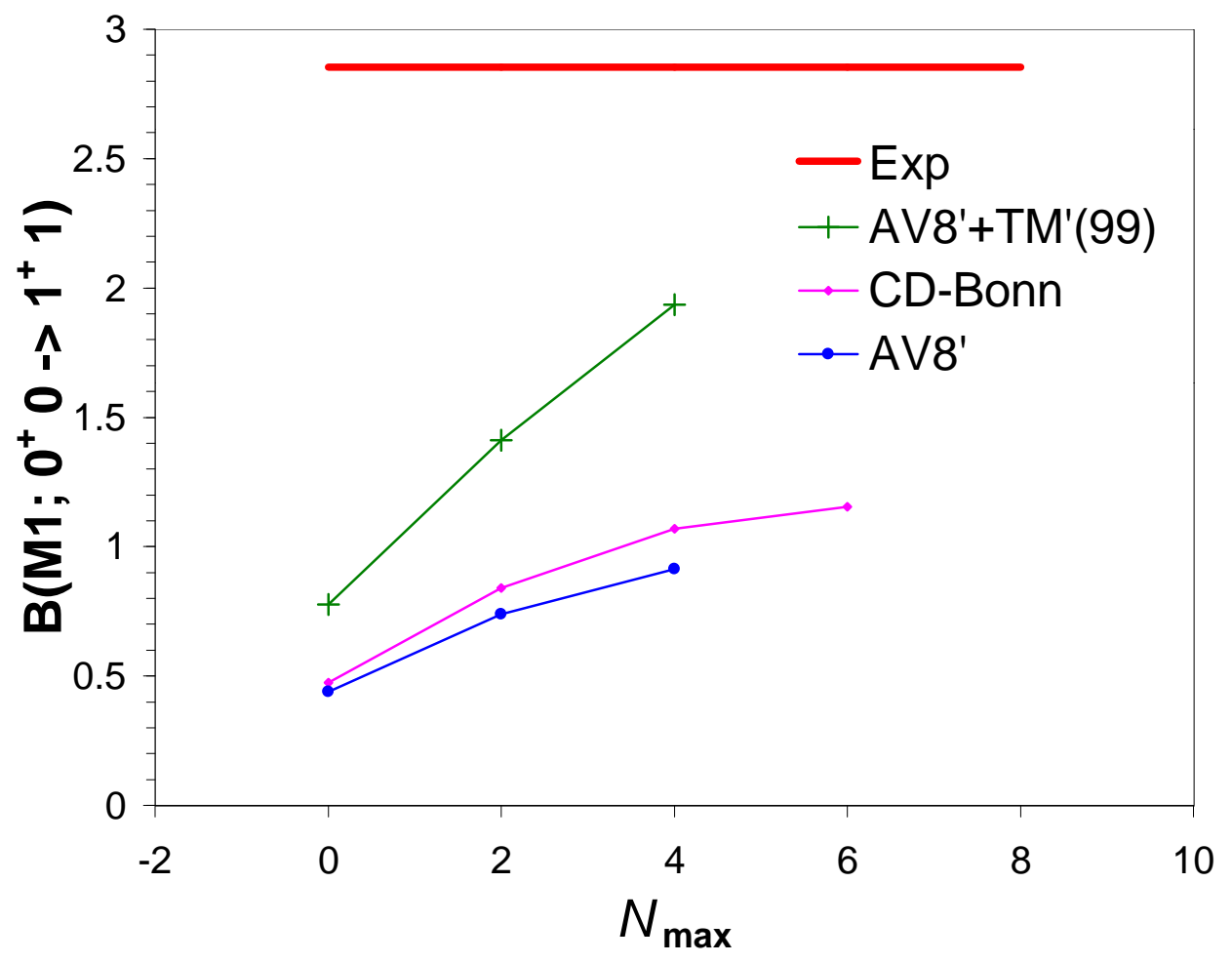

FIG. 2: $\mathrm{B}(\mathrm{M} 1)$ values, in $\mu_{N}^{2}$, of the $0^{+} 0 \rightarrow 1^{+} 1$ transition in ${ }^{12} \mathrm{C}$. For details see Table 


\begin{tabular}{c|c|c|c|cccc} 
& ${ }^{12} \mathrm{C}$ & $\mathrm{AV}^{\prime}+\mathrm{TM}^{\prime}(99)$ & $\mathrm{AV} 8^{\prime}$ & \multicolumn{5}{c}{$\mathrm{CD}-$ Bonn } \\
basis space & - & $4 \hbar \Omega$ & $4 \hbar \Omega$ & $6 \hbar \Omega$ & $4 \hbar \Omega$ & $2 \hbar \Omega$ & $0 \hbar \Omega$ \\
\hline$\left|E_{\mathrm{gs}}\right|[\mathrm{MeV}]$ & 92.162 & 91.963 & 85.944 & 85.630 & 88.518 & 92.375 & 104.947 \\
$Q_{2}\left[e \mathrm{fm}^{2}\right]$ & $+6(3)$ & 4.288 & 4.613 & 4.717 & 4.532 & 4.430 & 4.253 \\
\hline$E_{\mathrm{x}}\left(2^{+} 0\right)[\mathrm{MeV}]$ & 4.439 & 3.603 & 3.427 & 3.612 & 3.697 & 3.837 & 3.734 \\
$E_{\mathrm{x}}\left(1^{+} 0\right)[\mathrm{MeV}]$ & 12.710 & 11.280 & 13.926 & 13.930 & 14.140 & 14.524 & 13.866 \\
$E_{\mathrm{x}}\left(4^{+} 0\right)[\mathrm{MeV}]$ & 14.083 & 13.517 & 12.272 & 13.110 & 13.356 & 13.638 & 12.406 \\
$E_{\mathrm{x}}\left(1^{+} 1\right)[\mathrm{MeV}]$ & 15.110 & 16.221 & 16.364 & 16.064 & 16.165 & 16.291 & 15.290 \\
$E_{\mathrm{x}}\left(2^{+} 1\right)[\mathrm{MeV}]$ & 16.106 & 16.467 & 17.712 & 17.409 & 17.717 & 17.945 & 15.970 \\
$E_{\mathrm{x}}\left(0^{+} 1\right)[\mathrm{MeV}]$ & 17.760 & 17.116 & 16.213 & 16.534 & 16.619 & 16.493 & 14.698 \\
\hline $\mathrm{B}\left(\mathrm{E} 2 ; 2^{+} 0 \rightarrow 0^{+} 0\right)$ & $7.59(42)$ & 4.146 & 4.765 & 5.019 & 4.624 & 4.412 & 4.092 \\
$\mathrm{~B}\left(\mathrm{M} 1 ; 1^{+} 1 \rightarrow 0^{+} 0\right)$ & $0.951(20)$ & 0.645 & 0.305 & 0.384 & 0.355 & 0.280 & 0.158 \\
$\mathrm{~B}\left(\mathrm{E} 2 ; 2^{+} 1 \rightarrow 0^{+} 0\right)$ & $0.65(13)$ & 0.430 & 0.247 & 0.309 & 0.283 & 0.015 & 0.002
\end{tabular}

TABLE I: Experimental and calculated properties of ${ }^{12} \mathrm{C}$. The units are $e^{2} \mathrm{fm}^{4}\left(\mu_{N}^{2}\right)$ for B(E2) (B(M1)). Three-body effective interactions derived from the $\mathrm{AV}^{\prime}$ [14] and $\mathrm{AV}^{\prime}+\mathrm{TM}^{\prime}(99)$ and two-body effective interactions derived from the CD-Bonn [15] NN potential and a HO frequency of $\hbar \Omega=15 \mathrm{MeV}$ were used. The $\mathrm{TM}^{\prime}(99)$ parameters are given in Refs. 7, 10] with the cutoff set to $\Lambda=4.7$. The experimental values are from Ref. [16]. By extrapolating our results we predict the CD-Bonn ${ }^{12} \mathrm{C}$ binding energy to be $\approx 80 \pm 2 \mathrm{MeV}$.

\begin{tabular}{|c|c|c|c|c|c|}
\hline Interaction & CD-Bonn $2 \hbar \Omega$ & CD-Bonn $4 \hbar \Omega$ & CD-Bonn $6 \hbar \Omega$ & $\mathrm{AV}^{\prime}+\mathrm{TM}^{\prime}(99) 4 \hbar \Omega$ & experiment \\
\hline$\left(\nu_{e}, e^{-}\right)$ & 2.27 & 3.2 & 3.69 & 6.8 & $8.9 \pm 0.3 \pm 0.9[19]$ \\
\hline$\left(\nu_{\mu}, \mu^{-}\right)$ & 0.168 & 0.275 & 0.312 & 0.537 & $0.56 \pm 0.08 \pm 0.1[20]$ \\
\hline$\mu$-capture & 1.46 & 2.07 & 2.38 & 4.43 & $6.0 \pm 0.4[21]$ \\
\hline
\end{tabular}

TABLE II: Predicted weak interaction rates for the ${ }^{12} \mathrm{C} \rightarrow \mathrm{T}=11^{+}$transitions. The units are $10^{-42} \mathrm{~cm}^{2}$ for the $\left(\nu_{e}, e^{-}\right) \mathrm{DAR}$ cross section, $10^{-40} \mathrm{~cm}^{2}$ for $\left(\nu_{\mu}, \mu^{-}\right)$DIF cross section and $10^{3} \mathrm{sec}^{-1}$ for muon capture. 